\title{
The heterogeneity, distribution, and environmental associations of Borrelia burgdorferi sensu lato, the agent of Lyme borreliosis, in Scotland
}

\author{
Marianne C. James ${ }^{1,2+}$, Lucy Gilbert ${ }^{3}$, Alan S. Bowman ${ }^{1}$ and Ken J. Forbes ${ }^{2}$ \\ 1 Institute of Biological and Environmental Sciences, University of Aberdeen, Aberdeen, UK \\ 2 Division of Applied Medicine, University of Aberdeen, Aberdeen, UK \\ 3 James Hutton Institute, Aberdeen, UK
}

Edited by:

Rubén Bueno-Marí, University of Valencia, Spain

Reviewed by:

Jia Liu, Pfizer Inc., USA

Gaelle Marenne, Wellcome Trust

Sanger Institute, UK

*Correspondence:

Lucy Gilbert, James Hutton Institute Macaulay Drive, Craigiebuckler. Aberdeen AB15 8QH, UK e-mail: lucy.gilbert@hutton.ac.uk

${ }^{\dagger}$ Present address:

Marianne C. James, Food Standards Agency in Scotland, Aberdeen, UK
Lyme borreliosis is an emerging infectious human disease caused by the Borrelia burgdorferi sensu lato complex of bacteria with reported cases increasing in many areas of Europe and North America. To understand the drivers of disease risk and the distribution of symptoms, which may improve mitigation and diagnostics, here we characterize the genetics, distribution, and environmental associations of $B$. burgdorferi s.I. genospecies across Scotland. In Scotland, reported Lyme borreliosis cases have increased almost 10-fold since 2000 but the distribution of $B$. burgdorferi s.I. is so far unstudied. Using a large survey of over 2200 Ixodes ricinus tick samples collected from birds, mammals, and vegetation across 25 sites we identified four genospecies: Borrelia afzelii (48\%), Borrelia garinii (36\%), Borrelia valaisiana $(8 \%)$, and $B$. burgdorferi sensu stricto $(7 \%)$, and one mixed genospecies infection. Surprisingly, $90 \%$ of the sequence types were novel and, importantly, up to $14 \%$ of samples were mixed intra-genospecies co-infections, suggesting tick co-feeding, feeding on multiple hosts, or multiple infections in hosts. B. garinii (hosted by birds) was considerably more genetically diverse than $B$. afzelii (hosted by small mammals), as predicted since there are more species of birds than small mammals and birds can import strains from mainland Europe. Higher proportions of samples contained $B$. garinii and $B$. valaisiana in the west, while $B$. afzelii and $B$. garinii were significantly more associated with mixed/deciduous than with coniferous woodlands. This may relate to the abundance of transmission hosts in different regions and habitats. These data on the genetic heterogeneity within and between Borrelia genospecies are a first step to understand pathogen spread and could help explain the distribution of patient symptoms, which may aid local diagnosis. Understanding the environmental associations of the pathogens is critical for rational policy making for disease risk mitigation and land management.

Keywords: Ixodes ricinus, ticks, Lyme disease, MLST, PCR, genetics, allele, sequence type

\section{INTRODUCTION}

Lyme borreliosis is the most prevalent tick-borne human disease in the northern hemisphere, and is growing in incidence in Europe. For example, Scotland has seen an almost 10 -fold increase in reported cases since 2000 (1). The causative agent of Lyme borreliosis is Borrelia burgdorferi sensu lato, a complex of related spirochete bacteria comprising a suite of genospecies, which vary in pathogenicity and cause different symptoms. The global distribution of the genospecies differs between continents, for example, Borrelia afzelii and Borrelia garinii are found only in Europe, Borrelia carolinensis is found only in North America, and B. burgdorferi sensu stricto is found on both sides of the Atlantic (2).

There are currently at least 18 proposed and confirmed $B$. burgdorferi genospecies globally, which vary in their pathogenicity, reservoir host associations, and geographic distributions within and between countries (3-5). Three genospecies (B. burgdorferi s.s., B. garinii, and B. afzelii) are commonly reported to cause Lyme borreliosis and B. valaisiana and Borrelia lusitaniae may also be pathogenic (6-10).

Borrelia burgdorferi s.l. is transmitted by Ixodes ticks and, in most of Europe, including the UK, the principle vector is Ixodes ricinus. I. ricinus are generalist ecto-parasites, feeding on most terrestrial vertebrate species. However, each B. burgdorferi s.l. genospecies is specialized and associated with a particular host type. B. garinii and B. valaisiana are commonly found in birds, $B$. afzelii is associated with small mammals, and B. burgdorferi s.s. is associated with both birds and small mammals (11-13). We may therefore predict that genospecies prevalence varies with the relative abundance of these host types or, as a proxy, with different habitats that are associated with these hosts. In addition, within host types, some species are more effective at pathogen transmission than others, so we might predict within-genospecies genetic diversity to vary, for example, we might predict that $B$. garinii may have higher genetic diversity than $B$. afzelii because there are many 
more species of birds than small mammals. In addition, if genetic variation over space is driven by host movements [e.g., Ref. (14, 15)], we can predict that sequence types within genospecies are more closely related if they occur in closer geographic proximity; we can use spatial genetic variation to infer host movement behavior and in turn this helps us identify how each pathogen spreads over space.

Ixodes ricinus has three active stadia (larvae, nymphs, and adults), each of which requires a single blood meal. Unfed larvae are almost always uninfected because vertical transmission of $B$. burgdorferi s.l. from adult females to larvae is extremely rare (16). Despite the host specificity of each genospecies of B. burgdorferi s.l. and the general assumption that each tick stage feeds on only one host, co-infections of both "bird" and "mammal" genospecies can occur within a tick (3), although the frequency of such coinfections is less than expected (17). Such co-infections might suggest that the host specificity of genospecies is not absolute, or a tick stage may occasionally take more than one blood meal (from more than one host type), or through co-feeding [transmission from one tick to another without systemic host infection (18, 19)]. This interesting phenomenon warrants further research and one of our aims is to quantify the frequency and type of mixed infections in our studied I. ricinus populations.

The molecular characterization of B. burgdorferi genospecies and strains has been revolutionized by multi-loci sequence typing (MLST) and in this study we use the system developed by Margos and others (20) which has been shown to unambiguously delineate genospecies and establish evolutionary and geographic relationships. DNA can be directly amplified by polymerase chain reaction (PCR) from tick extracts and the amplicons sequenced without need for culture. The B. burgdorferi MLST website ${ }^{1}$ currently documents more than 1500 B. burgdorferi strains in the MLST database, currently comprising 572 sequence types from sites in Europe, North America, and Asia.

Previous MLST of studies of B. burgdorferi s.l. have been conducted at a continental scale $(15,20,21)$ or focused on a single genospecies $(20,22,23)$. Our study differs by employing a dense stratified survey to genetically and ecologically characterize the full suite of genospecies at a national scale. Characterizing the variety, distribution, and abundance of sequence types (i.e., the allelic profile) of B. burgdorferi s.l. in one country, especially linked to environmental information, will contribute to our understanding of the heterogeneous distribution and prevalence of genospecies, how the pathogens spread, identify the environmental risk factors and will also have implications for patient symptoms and diagnosis. We focused our B. burgdorferi s.l. survey on Scotland, where reported cases of Lyme borreliosis have increased almost 10-fold since the turn of the millenium, and where there have been very few previous studies (and no large-scale systematic surveys) of B. burgdorferi s.l. genospecies; (24) reported five B. afzelii samples and seven $B$. burgdorferi s.s. while (15) genotyped three B. burgdorferi samples from one Scottish site and found all to be B. afzelii.

This study therefore aimed to examine the phylogenetic population structure of Scottish B. burgdorferi s.l. by characterizing the genospecies, sequences types, and alleles and

\footnotetext{
${ }^{1}$ www.mlst.net
}

describing their spatial distribution across the country and identifying environmental and regional associations. This was to provide the first large-scale fundamental data on the Lyme borreliosis agents across this country and to gain insight into genetic mixing spatially across Scotland and with other countries (e.g., due to host movements). Furthermore, we were particularly interested in identifying mixed infections (both between genospecies and between sequence types within genospecies) within individual ticks, since this has implications both for patient symptoms and diagnosis and for our understanding of tick feeding behavior. We also aimed to correlate B. burgdorferi s.l. genospecies with environmental factors, which is of use in understanding the relationship between host communities and genospecies composition, and in assessing disease risk and mitigation options in different environments.

\section{MATERIALS AND METHODS FIELD COLLECTION OF TICKS}

Of the three life stages, nymphs are thought to pose the greatest risk to humans in terms of transmitting B. burgdorferi s.l.: questing larvae very rarely carry the pathogens (16), while adults are much less numerous than nymphs and are much larger and therefore more likely to be noticed and removed quickly. Indeed, (25) estimated that $82 \%$ of human tick bites from a forested area in England were from nymphs. Therefore, this study concentrated primarily on $I$. ricinus nymphs. Questing (host-seeking) nymphs were collected during blanket dragging surveys at 25 woodland sites across Scotland in the springs and summers of 2007-2008 [see Ref. (26)]. A $1 \mathrm{~m} \times 1 \mathrm{~m}$ square of blanket material was dragged for $10 \mathrm{~m}$ and all ticks counted and collected. At least 20 drags were conducted at each site in a semi-random fashion so as to cover the site in a representative way (26). At least 50 nymphs per site were screened for B. burgdorferi s.l. (see method below) including at least one nymph from each drag. Woodland was chosen because it is the habitat most often associated with high densities of a variety of tick species, both in Europe and North America [e.g., Ref. (27-30)] and the habitat most associated with acquiring Lyme borreliosis in Scotland (31). A broad geographic spread of sites ensured as much coverage over the country as possible, while collecting ticks from both semi-natural mixed/deciduous and conifer forests ensured that the main woodland habitat types (and therefore by proxy a range of host communities) were sampled. In addition, each site was associated with known cases of Lyme borreliosis (31).

As well as sampling questing nymphs from the 25 woodland sites, ticks were also removed from hosts. Passerine birds were trapped at one of the woodland sites by mist netting, under license issued by the British Trust for Ornithology during the spring and summer of 2008 [see Ref. (32)]. Feeding nymphs and larvae was removed from birds and stored in vials of $70 \%$ ethanol. Small mammals (wood mice Apodemus sylvaticus and bank voles Myodes glareolus) were trapped using longworth live traps, under license from Scottish Natural Heritage, at four of the woodland sites during the spring and summer of 2007 . Ticks were removed and stored in vials per animal in $70 \%$ ethanol.

\section{TICK POOLING FOR ANALYSIS}

Questing I. ricinus nymphs collected in 2007 were each homogenized and amplified individually by PCR. However, 
questing nymphs from 2008 were pooled in groups of five for processing. Our screening of nymphs collected in 2007 determined that the mean infection prevalence of B. burgdorferi s.l. was around $5 \%$, therefore, the probability of more than one positive tick occurring in a pool of five was only $2.3 \%$. Pooling was undertaken only for ticks collected at the same site on the same visit (and in the majority of cases from the same $10 \mathrm{~m} \times 1 \mathrm{~m}$ survey transect).

Feeding nymphal ticks removed from birds were PCR amplified individually (as feeding nymphs have now fed on two hosts). Feeding larval ticks removed from small mammals were pooled per animal. This was because transovarial transmission is thought to occur at very low frequencies (16) such that any larval ticks collected from one animal should have been exposed to $B$. burgdorferi s.l. present in that particular animal only. Between 2 and 28 larvae were removed from each small mammal and pooled for PCR (nymph ticks from mammals were collected in very small numbers and not assayed).

\section{BORRELIA BURGDORFERI S.L. SCREENING AND MLST}

DNA extraction was performed by mechanical destruction of the nymph and larval ticks by ammonia extraction $(32,33)$. A nested PCR for the 5S-23S rRNA IGS and visualization by agar electrophoresis were used to detect B. burgdorferi s. 1. (34). A positive control of B. lusitaniae (a genospecies not found in northern Europe) and a negative control were used in all PCRs so that it was possible to detect any cross contamination or false positives. MLST, which has been shown to unambiguously delineate genospecies (20), was used to type all positive samples at eight loci ( $c l p A, c l p X, p e p X, p y r G, r e c G, r p l B$, and $u v r A$ ) (after 20). Positive controls were also typed and comprised B. afzelii (strain VS461), B. garinii (20047), B. valaisiana (VS116), B. burgdorferi s.s. (B31), and B. lusitaniae (Poti B2). PCR products were sequenced in both directions using an ABI automated DNA sequencer.

Forward and reverse sequences were compared, aligned, and trimmed using Sequence Editor (version 1.0.3, Macintosh computers) and consensus sequences were assigned an allele number. New alleles were submitted to the MLST website ${ }^{1}$.

\section{PHYLOGENETIC ANALYSIS}

Phylogenetic trees were drawn using MEGA [Version 4.0.2 (35)], which was also used to calculate pair-wise genetic distances and nucleotide differences between sequences. We also used this analysis to test our prediction that B. garinii should have greater genetic diversity within Scotland than B. afzelii [because there are more birds than small mammal species in Scotland (and Europe) and birds can carry mainland European strains into Scotland].

To test our prediction that samples should be more genetically similar if they are from sites in close proximity (and genetically dissimilar if they are far apart), we used linear regression analysis to examine the relationship between the geographical distance (kilometer) between the collection sites and the molecular diversity in B. afzelii and B. garinii samples [as defined by the number of single nucleotide polymorphism differences, calculated in MEGA version 4.0.2 (35)]. We compared models that were fit using distance $(\text { distance })^{2}$, square root (distance), and log (distance +1$)$ in order to test for both linear and curvilinear relationships. We chose the best fit based on model outputs of $R$-squared and $F$ values and residual fits.

To estimate whether we sampled enough ticks to provide a full picture of alleles and sequence types over Scotland we used rarefaction curves. This is a standard method to gage the extent to which sampling achieves saturation coverage (i.e., all types of individual or species in a population are sampled) (36). By plotting the number of samples analyzed against the number of sequence types and alleles found for each of the eight loci, the shape of the curve indicates whether most of the alleles or sequence types have been found (i.e., when the curve plateaus because few additional alleles are being found) or whether there are many more to be discovered (i.e., the curve is still climbing steeply because more alleles are being found). Rarefaction curves were drawn in Analytical Rarefaction 1.3 (University of Georgia), both with data from this study and with B. burgdorferi s.s. data taken from Ref. (20).

\section{IDENTIFYING MIXED INFECTIONS}

To investigate which novel sequence types found may not be real sequence types but, instead, may be a result of mixed allele infections, we closely examined each allele combination in relation to sequence type using only those samples that were successfully analyzed at eight loci (Table S1 and Figure S1 in Supplementary Material). It was assumed that sequence types that are represented by more than one sample or have previously been added to pubMLST are genuine sequence types. It was also assumed that single locus variants of a sequence type with more than one occurrence were also genuine sequence types. In order to estimate the frequency of intra-genospecies co-infections, we examined $B$. afzelii genotypes because $B$. afzelii was the most frequently found genospecies (and, as the results show, had a very large proportion of novel sequence types).

As well as mixed infections, there are alternative hypotheses for novel and single-occurring sequence types. For example, they could arise as the result of homoplasy (the independent acquisition of the same nucleotide polymorphism in an unrelated lineage due to mutation) or horizontal gene transfer (recombination). We considered homoplasy to be unlikely if there was more than one single nucleotide polymorphism. We tested for horizontal gene transfer by using Clonal Frame (version 1.1) to examine the clonal relationships between sequence types and to estimate the recombination events, which may have disrupted inheritance (37).

\section{ENVIRONMENTAL ASSOCIATIONS}

Associations between genospecies and the environmental factors (geographical area, woodland type, and deer abundance) at each tick collection sites were examined. We chose two broad area categories: the Grampian region, which consists of the Cairngorms, Speyside, Deeside, and Moray in the northeastern quarter of Scotland, and all other sites further west of this region (generally characterized by a warmer and wetter climate than Grampian). Woodlands were categorized as either coniferous or mixed/deciduous. All of the coniferous category were commercial plantations generally consisting of Scots pine Pinus sylvestris or spruce Picea spp. with larch Larix decidua, apart from one conifer site, which was semi-natural old-growth Scots pine. The mixed/deciduous woodlands were semi-natural and consisted primarily of mixed birch 
Betula spp., rowan Sorbus aucuparia and sometimes oak Quercus spp., and beech Fagus sylvatica with occasional Scots pine. The index of abundance of deer was the number of groups of roe and red deer dung pellets counted per $10 \mathrm{~m} \times 1 \mathrm{~m}$ transect, averaged for each site (see also Ref. (26)).

To statistically test for associations of area and woodland type (categorical variables) with genospecies we used chi-square tests (a separate test each for area and for woodland type). These reveal differences in habitat and area between the proportions of each genospecies that make up the total number of samples tested at each site. To test for associations of deer abundance (continuous variable) with genospecies, we used a generalized linear mixed model including site as a random effect to account for multiple samples per site. Since the response variable was categorical the default model distribution was multinomial with a cumulative logit link. All statistical tests were conducted in SAS Version 9.3.

\section{RESULTS}

Of the more than $2200 \mathrm{I}$. ricinus tick samples assayed, 124 tested positive to B. burgdorferi s.l. and comprised 87 questing nymphs, 25 nymphs removed from birds, and 12 larvae from small mammals (Table 1). Fifty two samples were genotyped at all eight MLST loci and a phylogram of these sequence types was plotted along with most sequence types listed in the MLST database ${ }^{1}$ (Figure S1 in Supplementary Material).

There was no overlap (sharing) of alleles between any genospecies (either in our data set or in the pubMLST database), implying that samples could be classified into genospecies level without having to genotype at all eight loci, and this also means that identifying mixed genospecies infections is easy. Seventy five samples were genotyped successfully at fewer loci while still allowing the predominant genospecies to be determined. We were not able to amplify all loci for all genospecies. For example, in $B$.

Table 1 | Genospecies grouping of Scottish isolates by sample type and the number and type of samples processed using MLST at eight loci.

\begin{tabular}{lccc}
\hline Genospecies & $\begin{array}{c}\text { Questing } \\
\text { nymphs, } \boldsymbol{N}(\%)\end{array}$ & $\begin{array}{c}\text { Bird } \\
\text { nymphs, } \boldsymbol{N}(\%)\end{array}$ & $\begin{array}{c}\text { Mammal } \\
\text { larvae, } \boldsymbol{N} \text { (\%) }\end{array}$ \\
\hline B. afzelii & $42(48)$ & 0 & $12(100)$ \\
B. garinii & $31(36)$ & $24(96)$ & 0 \\
B. burgdorferi s. s. & $6(7)$ & 0 & 0 \\
B. valaisiana & $7(8)$ & $1(4)$ & 0 \\
B. lusitaniae & 0 & 0 & 0 \\
B. afzelii + B. garinii & $1(1)$ & 0 & 0 \\
No. samples & 87 & 25 & 12 \\
identifiable to & & & \\
genospecies & & 4 individuals & 8 pools of \\
No. samples & 40 (38 pools of \\
analyzed at 8 loci & $5+2$ individuals) & & $2-28$
\end{tabular}

Percentages show the proportion of all positive samples represented by the genospecies in question per tick type lquesting nymphs, nymphs attached to birds, and larvae attached to small mammals). valaisiana isolates, the $c l p A$ locus failed to amplify in six out of nine samples. This is likely attributable to sequence polymorphisms in the PCR primer oligo nucleotide sequences of these strains.

As defined by deep branching of phylogenetic trees, nonsharing of alleles and pair-wise genetic differences above the threshold described in Ref. (20) we identified that, of the total 124 positive tick samples from questing nymphs, nymphs from birds and larvae from small mammals, 55 (44\%) contained B. afzelii, 56 (45\%) contained B. garinii, 8 (6\%) were B. valaisiana, and 6 (5\%) were B. burgdorferi s.s. Of most importance to Lyme borreliosis risk in humans, out of the 87 questing nymph samples 43 (49\%) contained B. afzelii, 32 (36\%) contained B. garinii, 7 (8\%) were B. valaisiana, and 6 (7\%) were B. burgdorferi s.s. (Table 1).

\section{BORRELIA BURGDORFERI S.L. WITHIN-GENOSPECIES DIVERSITY}

Of the 52 samples typed at eight loci, there were 35 different sequence types, of which 28 were newly described to the MLST database. The most commonly occurring genospecies was B. afzelii and its sequence type ST263 accounted for 8/52 samples (15\%). The only other sequence types represented more than once in our study were ST287 (B. afzelii), ST168 (B. afzelii), ST327 (B. afzelii), and ST93 (B. garinii). All other sequence types were identified from only single samples in this study, although seven had been identified in previous studies ${ }^{1}$ : ST24 (B. burgdorferi s.s., found in France), ST82 (B. garinii, found in France and England), ST86 (B. garinii, found in France, England, Latvia, Russia, Austria, and Italy), ST88 (B. garinii, found in France and England), ST93 (B. garinii, found in France, Italy, and England), ST168 (B. afzelii found in Latvia), and ST205 (B. valaisiana, found in England).

The phylogenetic tree (Figure S1 in Supplementary Material) shows a greater variety of alleles, and with more branching, in $B$. garinii than in B. afzelii. In addition, the extent of nucleotide divergence at the loci was greatest for $B$. garinii, with $B$. garinii samples typically differing by $40-60$ bp across the eight loci compared to B. afzelii isolates which typically differed by only $1-15 \mathrm{bp}$.

Rarefaction analysis (Figure 1) indicated that for some of the eight loci, especially for B. afzelii, we detected most of the alleles in the population in Scotland (the curves plateau) while for other loci, especially for B. garinii, there are still several more alleles to be discovered (the curves are still climbing). For both B. afzelii and $B$. garinii, the curves for sequence type diversity did not plateau at all; indeed, for B. garinii the sequence type curve almost followed the $45^{\circ}$ line representing a new discovery for every sample analyzed.

There was a significant positive relationship between genetic and geographic distances for samples within the same genospecies, i.e., samples were more genetically different if they were collected further apart geographically, although the proportion of variation explained by the models ( $R$-squared values) was low (B. afzelii $F_{1,407}=22.9, p<0.001, R^{2}=51.0 \% ; B$. garinii $F_{1,209}=15.6$, $\left.p<0.0001, R^{2}=6.5 \%\right)$. The best fit models were those with simply distance (kilometer); those with (distance) ${ }^{2}$, square root (distance), or $\log ($ distance +1$)$ had poorer fit of the residuals and lower $R$-squared and $F$ values.

\section{MIXED INFECTIONS}

There was evidence of inter-genospecies mixed infections in one sample that clearly contained alleles originating from $B$. afzelii 


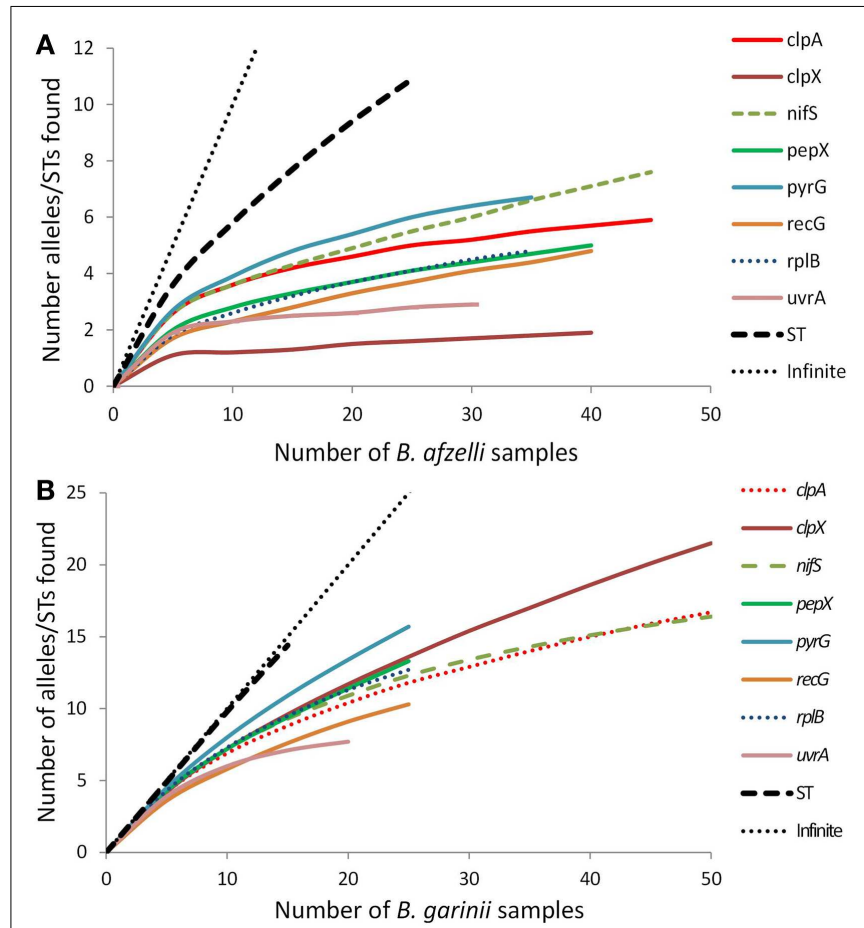

FIGURE 1 | Rarefaction curves for each of the eight $B$. burgdorferi s.I. loci and sequence types (STs) of (A) B. afzelii samples and (B) B. garinii samples analyzed from Scotland. Each graph also includes the $45^{\circ}$ line (labeled "infinite") which represents the trajectory that would occur if every new allele or sequence type found was new.

and B. garinii. This comprises $1.9 \%$ of the 52 samples tested across eight loci or $0.8 \%$ of the 124 total samples analyzed (including those successful at fewer than eight loci) and $1.1 \%$ of the 87 total samples of questing nymphs.

Close examination of the apparent 14 different sequence types found from the 29 B. afzelii samples (Table S1 in Supplementary Material) suggested the possible presence of mixed intragenospecies infections, deduced as follows. Five sequence types occurred more than once in the population (representing 20 samples) and so are plausibly genuine strains, while five sequence types (from five samples) differed at one of the two loci from the above and their different loci were the result of one or two nucleotide differences from the parental genotype so we considered these also to be plausibly genuine strains (for example ST292 has only one SNP difference in one allele from ST263). However, the remaining four sequence types (ST286, ST288, ST295, and ST326) were represented by only a single sample each and comprised either allele combinations found in other common sequence types (e.g., ST263) or two common sequence types plus a single nucleotide mutation in one of the alleles (Figure S1 in Supplementary Material). It can therefore be speculated that out of 29 B. afzelii samples, 25 isolates contained genuine sequence types while four apparent sequence types may actually be examples of intra-genospecies co-infection (i.e., harboring alleles from more than one B. afzelii sequence type). If so, this would represent a $14 \%$ incidence of intra-genospecies co-infection in the Scottish B. afzelii

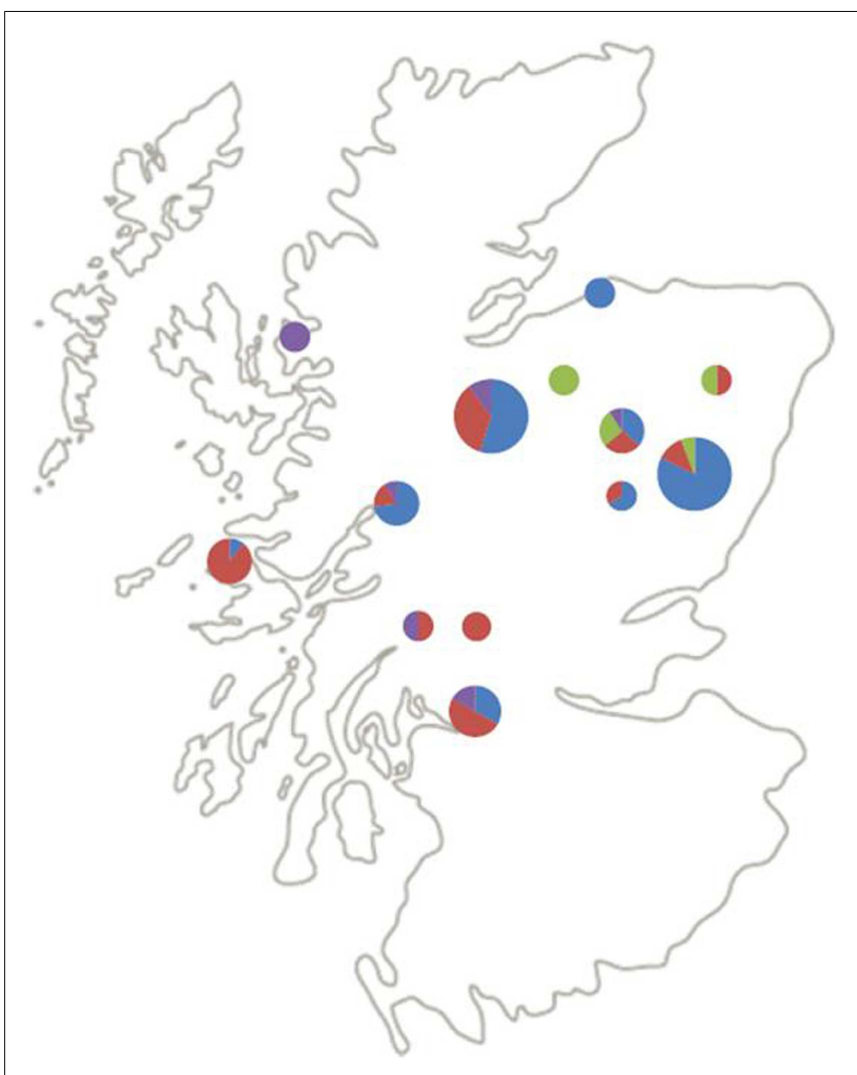

FIGURE 2 | Distribution and relative abundance of $\boldsymbol{B}$. burgdorferi s.l. genospecies across Scotland. Blue $=B$. afzelii, red $=B$. garinii, purple $=B$. valaisiana, green $=B$. burgdorferi s.s. The size of the pie charts indicates the number of samples genotyped at that site (1-5, 6-10, and 11-20 samples for small, medium, and large pie charts, respectively).

population. However, from only 29 samples, the confidence intervals are wide (upper and lower confidence intervals $=30.6$ and $5.5 \%$, respectively).

Homoplasy as a mechanism for the apparent novel sequence types seems unlikely since only two of the four appeared to contain a single nucleotide mutation and in only one of the mixed alleles. There was no evidence that horizontal gene transfer (recombination) is the explanation for these apparent sequence types because no recombination events were detected, either using only our Scottish samples or using a combination of Scottish samples and other sequence types listed in the MSLT database.

\section{ENVIRONMENTAL ASSOCIATIONS}

Figure 2 shows the geographical distribution of the genospecies of the 87 questing nymphs from the 13 (of the original 25) field sites from which positive $B$. burgdorferi s.l. samples were successfully identified to genospecies. Six sites were coniferous and seven seminatural mixed or deciduous woodlands. B. afzelii and B. garinii, the most frequent genospecies, seem to be spread over most of Scotland. B. afzelii was detected in 7 of the 13 sites and B. garinii in 11 of the 13 sites (Table 2). Of the less frequently recorded genospecies, B. valaisiana had a broad geographic spread and occurred in 5 of the 13 sites whereas B. burgdorferi s.s. was recorded only in 4 sites, 
Table 2 | Genospecies of B. Burgdorferi s.l. and sample size of positive samples from questing nymphs at each site.

\begin{tabular}{|c|c|c|c|c|c|c|c|}
\hline Site & Habitat & $\begin{array}{l}\text { Deer } \\
\text { index }\end{array}$ & B. garinii & B. afzelii & B. valaisiana & $\begin{array}{l}\text { B. burgdorferi } \\
\text { s.s. }\end{array}$ & $\begin{array}{l}\text { Total } \\
\text { B. burgdorferi s.I. }\end{array}$ \\
\hline QC & Conifer & 0.94 & $3(27 \%)$ & $4(36 \%)$ & $1(9 \%)$ & $3(27 \%)$ & 11 \\
\hline $\mathrm{GM}$ & Conifer & 0 & $2(50 \%)$ & $0(0 \%)$ & $2(50 \%)$ & $0(0 \%)$ & 4 \\
\hline BM & Conifer & 0.61 & $1(33 \%)$ & $2(67 \%)$ & $0(0 \%)$ & $0(0 \%)$ & 3 \\
\hline $\mathrm{CB}$ & Conifer & 0.35 & $0(0 \%)$ & $3(100 \%)$ & $0(0 \%)$ & $0(0 \%)$ & 3 \\
\hline AP & Conifer & 0 & $0(0 \%)$ & $0(0 \%)$ & $1(100 \%)$ & $0(0 \%)$ & 1 \\
\hline DR & Mixed & 0.01 & 7 (35\%) & 11 (55\%) & $2(10 \%)$ & $0(0 \%)$ & 20 \\
\hline $\mathrm{FZ}$ & Mixed & 0 & $2(12 \%)$ & $14(82 \%)$ & $0(0 \%)$ & $1(6 \%)$ & 17 \\
\hline LA & Mixed & 0 & $2(18 \%)$ & $8(73 \%)$ & $1(9 \%)$ & $0(0 \%)$ & 11 \\
\hline LV & Mixed & 0.06 & $1(50 \%)$ & $0(0 \%)$ & $0(0 \%)$ & $1(50 \%)$ & 2 \\
\hline All & & & 32 (35\%) & 45 (49\%) & $8(9 \%)$ & $7(8 \%)$ & 92 \\
\hline
\end{tabular}

Deer index is the number of pellet groups per $10 \mathrm{~m} \times 1 \mathrm{~m}$ transect averaged per site.

all in north-east Scotland (although with only six samples from questing nymphs in this study it may yet be recorded elsewhere if more samples are collected in future studies; Table 2).

There was a significant difference between genospecies and the two broad area categories $\left(\chi^{2}=18.8, \mathrm{df}=3, p=0.0003\right)$. $B$. garinii and $B$. valaisiana comprised the highest proportions of samples in the western half of Scotland while B. burgdorferi s.s. was most associated with the Grampian region (Figure 3). There was also a significant difference between genospecies and woodland type $\left(\chi^{2}=13.9, \mathrm{df}=3, p=0.0031\right)$. B. afzelii and B. garinii were more likely to be found in mixed/deciduous than in conifer forests and B. burgdorferi s.s., while occurring at only low prevalences, had a tendency to be found more frequently in coniferous forest (Figure 3). There was no evidence for a significant association between genospecies and the deer abundance at each site $\left(F_{1,72}=0.53, p=0.468\right)$.

\section{DISCUSSION}

The overall B. burgdorferi s.l. prevalence of 5.6\% (range 1-14\%) in questing nymphs [see also Ref. (26)] is similar to that found in a previous UK study (15) that found 5 and $8 \%$ prevalence in $2006-$ 2007 and 2008-2009, respectively (range 0-12\%) in ticks collected from sites mainly in England. This is a lower prevalence than that found in many countries in continental Europe [e.g., Ref. $(3,15)]$.

Of the B. burgdorferi s.l. positive questing nymphs, $49 \%$ were B. afzelii, $36 \%$ were B. garinii, $8 \%$ were B. valaisiana, and $7 \%$ were B. burgdorferi s.s. The finding that the most common genospecies in Scotland is B. afzelii [see also Ref. (24) which found that almost half of its 12 positive I. ricinus from the Scottish highlands carried B. afzelii] while $B$. valaisiana is relatively rare is interesting, because a quite different pattern has been found in England where B. afzelii is less common while B. garinii and B. valaisiana (the two birdassociated genospecies) seem to be predominant [e.g., Ref. (38)],
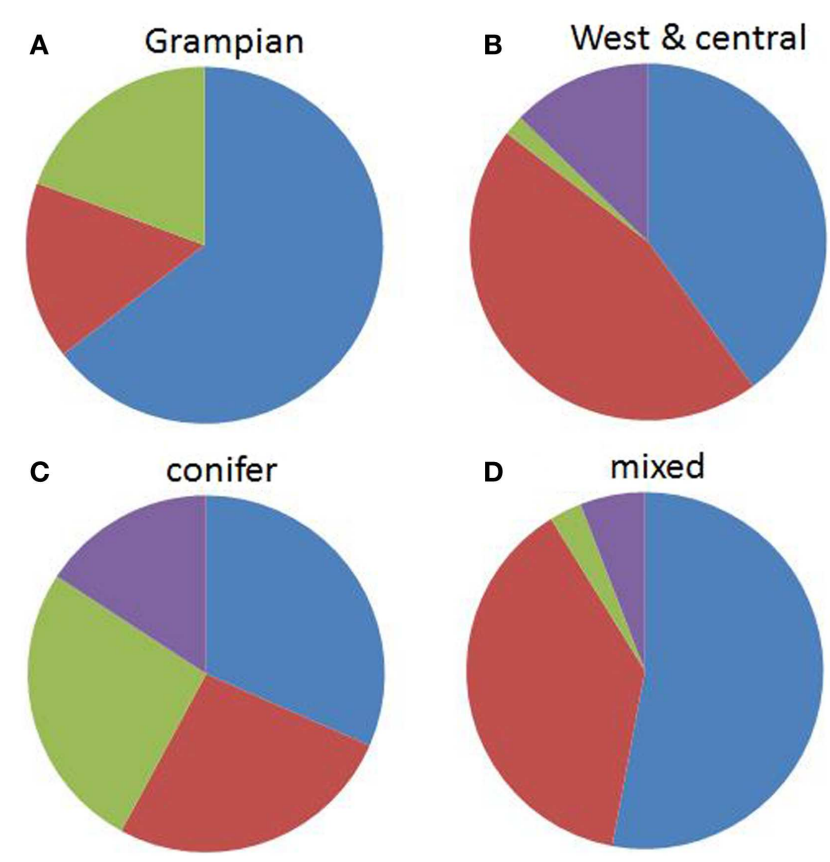

FIGURE 3 |The proportions of Borrelia burgdorferi s.I. genospecies by area: (A) west and central Scotland and (B) the Grampian Region of Scotland (the Northeast and the Cairngorms) and by habitat: (C) conifer forest and (D) mixed/deciduous woodlands, averaged over 13 (6 coniferous, $7 \mathrm{mixed} /$ deciduous) sites across Scotland. Blue $=B$. afzelii, red = B. garinii, purple $=B$. valaisiana, and green $=B$. burgdorferi s.s.

although Ref. (15) found B. afzelii at around half of their English sites. The reasons for any difference in genospecies predominance between Scotland and the rest of the British Isles warrants further 
research. The abundance of B. afzelii in Scotland is more similar to the situation in continental Europe where it is also common and often the dominant genospecies [e.g., Ref. $(3,39,40)]$.

Corroborating current knowledge of genospecies specificity [e.g., Ref. (38)], we found that the sole genospecies found in larval ticks removed from small mammals (wood mice and bank voles) was $B$. afzelii while, as expected, B. garinii was the dominant genospecies found in nymphs removed from passerine birds (and one B. valaisiana sample from a bird).

\section{WITHIN-GENOSPECIES DIVERSITY}

As we predicted from the concept that the spatial distribution of genetic relatedness is driven by local host movements, for both $B$. afzelii and B. garinii, we found a positive relationship between geographical distance (kilometer separating field sites) and genetic distance (single nucleotide polymorphism differences) between pairs of samples within genospecies, i.e., alleles and sequence types are more different the further apart they are geographically. However, the most abundant strain type (B. afzelii ST263) occurs widely across Scotland (up to $110 \mathrm{~km}$ in this study), and could perhaps, therefore, be an ancestral strain. B. garinii was considerably more genetically diverse than B. afzelii (that was dominated by ST263). This is consistent with our predictions based on higher number of bird species than small mammal species and the higher frequency of non-local alleles being brought into Scotland from other countries by migrating birds. We found that all (apart from one, previously identified from Germany) of the B. afzelii sequence types we identified from Scotland were novel. This helps corroborate the proposal of Ref. (15) (stemming from previous limited data on Scottish Borrelia burgdorferi s.l.) that there should be a clear distinction between English and Scottish B. afzelii due to limited movement of small mammals between England and Scotland [see Ref. (41)]. In contrast to B. afzelii, several B. garinii sequence types that we identified in Scotland had previously been identified from France and England, suggestive of greater host (bird) movement between these countries. A high degree of diversity in B. garinii samples has also been noted in Ref. (42), among others, who attributed this to their bird host's large migration ranges.

\section{MIXED INFECTIONS}

The identification of mixed samples can be influenced by the extent of horizontal gene transfer events, which will decrease the observed clonality of the species. In the case of B. burgdorferi s.l., however, there is no evidence to support horizontal gene transfer of chromosomal genes and, certainly between genospecies, the deep branching, and absence of pan-alleles argues against horizontal gene transfer. We found only one sample clearly exhibiting alleles from two genospecies, which is most likely to be a result of mixed-genospecies infections within a tick. This is a frequency of $1-2 \%$ (depending on whether the total number of 124 samples is used or only those 52 samples analyzed at all eight loci or including only questing nymphs). This is much lower than many other areas: a meta-analysis in Ref. (3) of over 100 articles citing the infection prevalences in 112,579 questing ticks from 24 countries across Europe found that the occurrence of inter-genospecies coinfection in Ixodid nymphs was, overall, $12.1 \%$, while as many as $64 \%$ of questing nymphs were co-infected in Denmark (43).
Assuming that transovarial transmission of B. burgdorferi is absent or rare such that unfed larvae are uninfected $(16,44)$ outlined three potential mechanisms for an unfed nymph to be co-infected with more than one genospecies: (i) through transmission by co-feeding (feeding in close proximity to a tick infected with a different genospecies to that in the host); (ii) through being unable to complete a full blood meal from one host (e.g., dislodged before repletion) and so feeding from a second host containing a different B. burgdorferi s.l. genospecies; or (iii) through feeding from a single host that carried more than one genospecies, perhaps because of a compromised immune system. Most mixed infections in Europe are B. garinii mixed with B. valaisiana (3) (i.e., the two genospecies associated with birds). It is noteworthy, therefore, that our two mixed genospecies samples contained alleles from B. afzelii and B. garinii, the small mammal- and the bird-associated genospecies respectively. Therefore, it is unlikely that our mixed infection resulted from a host infected with both genospecies. However, it could have resulted from the tick having an incomplete feed on a B. afzelii infected small mammal followed by a further feed on a $B$. garinii infected bird (or vice versa). It could also have potentially resulted from co-feeding: the tick attached to a bird or small mammal infected with one pathogen while feeding in close proximity to a nymph that was infected with the second pathogen.

While mixed infections of different genospecies are relatively easy to identify in samples and are now well documented [e.g., Ref. $(3,15)]$, it may be more likely for a tick to be co-infected with different strains of the same genospecies (each with a different sequence type), although it is much less easy to identify. This phenomenon has been previously reported: $39 \%$ of adult Ixodes scapularis ticks from North America were infected with more than one genotype of B. burgdorferi s.s. (45). From close examination of the novel sequence types we found, we estimated that the incidence of intra-genospecies co-infection is around $14 \%$ in the Scottish $B$. afzelii population. We also considered the alternative explanations for these four apparent sequence types. The allele combinations ruled out vertical inheritance. Homoplasy is unlikely since several of the shared alleles harbor more than one single nucleotide polymorphism compared to the putative ancestral strain in several cases. In addition, it has been reported as an unlikely event in the $B$. burgdorferi s.l. genome and nearly non-existent in the chromosome [reviewed in Ref. (46)]. At an environmental scale, there is no evidence of horizontal gene transfer in the IGS locus, but the ospC locus showed evidence of intragenic recombination (47). It would appear that recombination events are possible within the $B$. burgdorferi s.l. genome, but they are rare and limited to particular genes (e.g., ospC). ospC may be subject to recombination in a way that other genes are not due to its role in the immune system (unlike the selectively neutral housekeeping genes of MLST) (48).

Our finding of commonly occurring mixed within-genospecies co-infections is important also because it suggests that the MLST database may inadvertently contain examples of mixed intragenospecies co-infection. Detecting and excluding these samples could be overcome only by culturing colonies from ticks and selecting individual colonies or by using detection methods, which can pick up multiple genospecies in a sample. Unless samples in the MLST database contain sequence types from individual colony 
cultures (the majority are not, as they are from field collected whole ticks), then the database may contain many of these intragenospecies mixed sequence types and should therefore be treated with caution.

More work is required to examine this phenomenon of mixed infections, for example by the cultural separation of samples with mixed chromatograms or forensic methods such as designing PCR probes to identify individual genospecies or even specific alleles. This may also help answer whether intra-genospecies co-infection is more common within certain genospecies. It perhaps would be more likely for intra-genospecies co-infection to occur in $B$. garinii, as there is more diversity of allele numbers.

\section{ENVIRONMENTAL ASSOCIATIONS}

Borrelia afzelii was found relatively evenly throughout Scotland whereas B. burgdorferi s.s. was found only around the Grampian region of north east Scotland (albeit detected in only nine samples). B. garinii and B. valaisiana were widely spread across Scotland although, statistically, they occurred at higher proportions in the warmer and wetter western areas of Scotland. These distributional differences are likely to be associated with differences in relative host abundances, which are generally driven by habitat which in turn is affected by both climate and anthropogenic land management goals. Four of the six (67\%) Grampian sites were coniferous, compared with two of the seven (29\%) western sites but within each of our two very basic woodland categories are many sub-categories of habitat such as lowland deciduous, upland birch, Atlantic oak, juniper scrub, ancient pine, and commercial plantation. While the number of sites we originally sampled was 25 , many more would be needed to statistically test genospecies associations with finer habitat categories. However, using our two basic woodland types, B. garinii and B. afzelii more likely to be found in deciduous forests while higher proportions of $B$. burgdorferi s.s. were found in coniferous forest. This is likely to reflect the differences in relative abundance of host types between habitats: B. garinii and B. afzelii are associated with birds and small mammals, respectively, and semi-natural mixed or deciduous woodlands are generally associated with higher abundance and biodiversity of both birds and small mammals than are conifer plantations. However, it is unclear why B. burgdorferi s.s. should occur more frequently in coniferous woodlands, since previous studies suggest that the key reservoir hosts for B. burgdorferi s.s. are, as for B. afzelii, small mammals [e.g., Ref. (49, 50)]. Given the low number of $B$. burgdorferi s.s. positive samples we found in Scotland, this could be a statistical artifact, but further work is required to identify the key reservoir host for B. burgdorferi s.s. in Scotland and its relative abundance between habitats. Given that the spatial distribution of B. burgdorferi s.s. seems to be restricted to the Grampian Region and Speyside, and there may be an association with coniferous forests, we can speculate that red squirrels Sciurus vulgaris (that are also more abundant in conifer forests in this region than many other parts of Scotland) could be important B. burgdorferi s.s. in Scotland. Indeed, B. burgdorferi s.s. is prevalent in red squirrels in Switzerland and red squirrels can transmit B. burgdorferi s.s. to feeding ticks (51). Similarly, associations have been found between B. burgdorferi s.s. and western gray squirrels Sciurus griseus in California (52).

\section{CONCLUSION}

This large-scale intensive analysis of more than 2000 I. ricinus tick samples from over $120010 \mathrm{~m} \times 1 \mathrm{~m}$ transect surveys, and from birds and small mammals, at 25 sites has provided the first comprehensive analysis of the B. burgdorferi s.l. genospecies present in Scotland. That the most prevalent genospecies was B. afzelii was surprising as it has been postulated to be rare in the United Kingdom $(2,15)$. We found much lower inter-genospecies coinfections (1\%) than found in other countries but, importantly, we found frequent intra-genospecies co-infections (14\% of B. afzelii), suggesting co-feeding ticks, ticks feeding on multiple hosts, or multiple infections within hosts. Our findings that genetic and geographic distances are positively correlated and the differences in intra-genospecies genetic diversity can help us understand how, and from where, each pathogen spreads spatially over time. We speculate that red squirrels may be an important reservoir host for B. burgdorferi s.s. in northeastern Scotland, from circumstantial evidence of its regional and habitat associations, as well as previous evidence from Switzerland. The association between some genospecies and geographic area could be useful to practitioners in diagnostics, since each genospecies varies in the symptoms caused, especially if future work can determine the pathogenicity of different local strains. By examining the spatial patterns of genospecies and strain types in many countries, and linking this to their pathogenicity, it may become possible to understand the heterogeneous spatial distributions of genospecies, disease risk, and patient symptoms across more globally.

\section{ACKNOWLEDGMENTS}

Genospecies controls were obtained from the laboratory of Dr. Muriel Cornet at the Institut Pasteur, Paris. We thank Bob Furness for collecting ticks from passerine birds, Steph Vollmer for processing the samples from one site, E. Packer, A. Wiebe, J. Low, E. Stephen, and J. Arthur for help collecting ticks, Kenny Raey for laboratory assistance, and Jackie Potts for statistical advice. Marianne C. James was funded by a Biotechnology and Biological Sciences Research Council (BBSRC) Doctoral Training Grant with CASE support from the Macaulay Development Trust awarded to Alan S. Bowman and Lucy Gilbert. Lucy Gilbert was supported by the Scottish Government's Rural and Environment Science and Analytical Services Division (RESAS).

\section{SUPPLEMENTARY MATERIAL}

The Supplementary Material for this article can be found online at http://www.frontiersin.org/Journal/10.3389/fpubh.2014. 00129/abstract

\section{REFERENCES}

1. Health Protection Scotland. (2014). Available from: http://www.documents.hps. scot.nhs.uk/giz/10-year-tables/lyme.pdf

2. Kurtenbach K, Hanincová K, Tsao JI, Margos G, Fish D, Ogden NH. Fundamental processes in the evolutionary ecology of Lyme borreliosis. Nat Rev Microbiol (2006) 4:660-9. doi:10.1038/nrmicro1475

3. Rauter C, Hartung T. Prevalence of Borrelia burgdorferi sensu lato genospecies in Ixodes ricinus ticks in Europe: a metaanalysis. Appl Environ Microbiol (2005) 71:7203-16. doi:10.1128/AEM.71.11.7203-7216.2005

4. Margos G, Hojgaard A, Lane RS, Cornet M, Fingerle V, Rudenko N, et al. Multilocus sequence analysis of Borrelia bissettii strains from North America reveals a 
new Borrelia species, Borrelia kurtenbachii. Ticks Tick Borne Dis (2010) 1:151-8. doi:10.1016/j.ttbdis.2010.09.002

5. Rudenko N, Golovchenko M, Grubhoffer L, Oliver HJ Jr. Updates on Borrelia burgdorferi sensu lato complex with respect to public health. Ticks Tick Borne Dis (2012) 2:123-8. doi:10.1016/j.ttbdis.2011.04.002

6. Assous MV, Postic D, Paul G, Nevot P, Baranton G. Western blot analysis of sera from Lyme borreliosis patients according to the genomic species of the Borrelia strains used as antigens. Eur J Clin Microbiol Infect Dis (1993) 12:261-8. doi:10.1007/BF01967256

7. Van Dam AP, Kuiper H, Vos K, Widjojokusumo A, De Jongh BM, Spanjaard L, et al. Different genospecies of Borrelia burgdorferi are associated with distinct clinical manifestations of Lyme borreliosis. Clin Infect Dis (1993) 17:708-17. doi:10.1093/clinids/17.4.708

8. Collares-Pereira M, Couceiro S, Franca I, Kurtenbach K, Schäfer SM, Vitorino L, et al. First isolation of Borrelia lusitaniae from a human patient. J Clin Microbiol (2004) 42:1316-8. doi:10.1128/JCM.42.3.1316-1318.2004

9. Busch U, Hizo-Teufel C, Boehmer R, Fingerle V, Nitschko H, Wilske B, et al. Three species of Borrelia burgdorferi sensu lato (B. burgdorferi sensu stricto, $B$. afzelii, and B. garinii) identified from cerebrospinal fluid isolates by pulsed-field gel electrophoresis and PCR. J Clin Microbiol (1996) 34:1072-8.

10. Rijpkema SGT, Tazelaar DJ, Molkenboer MJCH, Noordhoek GT, Plantinga G, Schouls LM, et al. Detection of Borrelia afzelii, Borrelia burgdorferi sensu stricto, Borrelia garinii and group VS116 by PCR in skin biopsies of patients with erythema migrans and acrodermatitis chronica atrophicans. Clin Microbiol Infect (1997) 3:109-16. doi:10.1111/j.1469-0691.1997.tb00259.x

11. Richter D, Spielman A, Komar N, Matuschka F. Competence of American robins as reservoir hosts for Lyme disease spirochetes. Emerg Infect Dis (2000) 6:133-8. doi:10.3201/eid0602.000205

12. Hanincova K, Schäfer SM, Etti S, Sewell H, Taragelová V, Ziak D, et al. Association of Borrelia afzelii with rodents in Europe. Parasitology (2003) 126:11-20. doi:10.1017/S0031182002002548

13. Hanincová K, Taragelová V, Koci J, Schäfer SM, Hails R, Ullmann AJ, et al. Association of Borrelia garinii and B. valaisiana with songbirds in Slovakia. Appl Environ Microbiol (2003) 69:2825-30. doi:10.1128/AEM.69.5.2825-2830.2003

14. Kurtenbach K, De Michelis S, Sewell HS, Etti S, Schäfer SM, Holmes E, et al. The key roles of selection and migration in the ecology of Lyme borreliosis. Int J Med Microbiol (2002) 291(Suppl 33):152-4. doi:10.1016/S1438-4221(02)80029-7

15. Vollmer SA, Bormane A, Dinnis RE, Seelig F, Dobson AD, Aanensen DM, et al. Host migration impacts on the phylogeography of Lyme borreliosis spirochaete species in Europe. Environ Microbiol (2011) 13:184-92. doi:10.1111/j.14622920.2010.02319.x

16. Hubálek Z, Halouzka J. Prevalence rates of Borrelia burgdorferi sensu lato in host-seeking Ixodes ricinus ticks in Europe. Parasitol Res (1998) 84:167-72. doi: $10.1007 /$ s004360050378

17. Herrmann C, Gern L, Voordouw MJ. Species co-occurrence patterns among Lyme borreliosis pathogens in the tick vector Ixodes ricinus. Appl Environ Microbiol (2013) 79:7273-80. doi:10.1128/AEM.02158-13

18. Labuda M, Jones LD, Williams T, Danielova V, Nuttall PA. Efficient transmission of tick-borne encephalitis virus between co-feeding ticks. J Med Entomol (1993) 30:295-9.

19. Ogden NH, Nuttall PA, Randolph SE. Natural Lyme disease cycles maintained via sheep by co-feeding ticks. Parasitology (1997) 115:591-9. doi:10.1017/ S0031182097001868

20. Margos G, Gatewood AG, Aanensen DM, Hanincová K, Terekhova D, Vollmer SA, et al. MLST of housekeeping genes captures geographic population structure and suggests a European origin of Borrelia burgdorferi. Proc Nat Acad Sci U $S$ A (2008) 105:8730-5. doi:10.1073/pnas.0800323105

21. Margos G, Vollmer SA, Cornet M, Garnier M, Fingerle V, Wilske B, et al. A new Borrelia species defined by multilocus sequence analysis of housekeeping genes. Appl Environ Microbiol (2009) 75:5410-6. doi:10.1128/AEM.00116-09

22. Victorino LR, Margos G, Feil EJ, Collares-Pereira M, Zé-Zé L, Kurtenbach K. Fine-scale phylogeographic structure of Borrelia lusitaniae revealed by multilocus sequence typing. PLoS One (2008) 3:e4002. doi:10.1371/journal.pone. 0004002

23. Gatewood AG, Liebman KA, Vourc'h G, Bunikis J, Hamer SA, Cortinas R, et al. Climate and tick seasonality are predictors of Borrelia burgdorferi genotype distribution. Appl Environ Microbiol (2009) 75:2476-83. doi:10.1128/AEM. 02633-08
24. Ling CL, Joss AWL, Davidson MM, Ho-Yen DO. Identification of different Borrelia burgdorferi genomic groups from Scottish ticks. Mol Pathol (2000) 53:94-8. doi: $10.1136 / \mathrm{mp} .53 .2 .94$

25. Robertson JN, Gray JS, Stewart P. Tick bite and Lyme borreliosis risk at a recreational site in England. Eur J Epidemiol (2000) 16:647-52. doi:10.1023/ A:1007615109273

26. James MC, Bowman AS, Forbes KJ, Lewis F, McLeod JE, Gilbert L. Environmental determinants of Ixodes ricinus ticks and the incidence of Borrelia burgdorferi sensu lato, the agent of Lyme borreliosis, in Scotland. Parasitology (2013) 140:237-46. doi:10.1017/S003118201200145X

27. Bertrand MR, Wilson ML. Microclimate-dependent survival of unfed adult Ixodes scapularis (Acari: ixodidae) in nature: life cycle and study design implications. J Med Entomol (1996) 33:619-27.

28. Lindsay LR, Barker IK, Surgeoner GA, McEwen SA, Gillespie TJ, Addison EM. Survival and development of the different life stages of Ixodes scapularis (Acari: ixodidae) held within four habitats on Long Point, Ontario, Canada. J Med Entomol (1998) 35:189-99.

29. Estrada-Peña A. Distribution, abundance, and habitat preferences of Ixodes ricinus (Acari: Ixodidae) in northern Spain. J Med Entomol (2001) 38:361-70. doi:10.1603/0022-2585-38.3.361

30. Barandika JF, Berriatua E, Barral M, Juste RA, Anda P, García-Pérez AL. Risk factors associated with ixodid tick species distributions in the Basque region in Spain. Med Vet Entomol (2006) 20:177-88. doi:10.1111/j.1365-2915.2006. 00619.x

31. James MC. The Ecology, Genetic Diversity and Epidemiology of Lyme Borreliosis in Scotland. Ph.D. thesis, Aberdeen: University of Aberdeen (2010).

32. James MC, Furness RW, Bowman AS, Forbes KJ, Gilbert L. The importance of passerine birds as tick hosts and in the transmission of Borrelia burgdorferi, the agent of Lyme disease. A case study from Scotland. Ibis (2011) 153:293-302. doi:10.1111/j.1474-919X.2011.01111.x

33. Guy EC, Stanek G. Detection of Borrelia burgdorferi in patients with Lyme disease by the polymerase chain reaction. J Clin Pathol (1991) 44:610-1. doi:10.1136/jcp.44.7.610

34. Rijpkema SGT, Molkenboer MJCH, Schouls LM, Jongejan F, Schellekens JFP. Simultaneous detection and genotyping of three genomic groups of Borrelia burgdorferi sensu lato in Dutch Ixodes ricinus ticks by characterization of the amplified intergenic spacer region between $5 \mathrm{~S}$ and 23S rRNA genes. JClin Microbiol (1995) 33:3091-5.

35. Tamura K, Dudley J, Nei M, Kumar S. MEGA4: molecular evolutionary genetics analysis (MEGA) software version 4.0. Mol Biol Evol (2007) 24:1596-9. doi:10.1093/molbev/msm092

36. Raup DM. Taxonomic diversity estimation using rarefaction. Paleobiology (1975) 1:333-42.

37. Didelot X, Falush D. Inference of bacterial microevolution using multilocus sequence data. Genetics (2007) 175:1251-66. doi:10.1534/genetics.106. 063305

38. Kurtenbach K, Peacey M, Rijpkema SGT, Hoodless AN, Nuttall PA, Randolph SE. Differential transmission of the genospecies of Borrelia burgdorferi sensu lato by game birds and small rodents in England. Appl Environ Microbiol (1998) 64:1169-74.

39. Etti S, Hails R, Schäfer SM, De Michelis S, Sewell H-S, Bormane A, et al. Habitatspecific diversity of Borrelia burgdorferi sensu lato in Europe, exemplified by data from Latvia. Appl Environ Microbiol (2003) 69:3008-10. doi:10.1128/AEM.69. 5.3008-3010.2003

40. Van Overbeek L, Gassner F, Lombaers van der Plas C, Kastelein P, Nunesda Rocha U, Takken W. Diversity of Ixodes ricinus tick-associated bacterial communities from different forests. FEMS Microbiol Ecol (2008) 66:72-84. doi:10.1111/j.1574-6941.2008.00468.x

41. Searle JB, Kotlík P, Rambau RV, Marková S, Herman JS, McDevitt AD. The Celtic fringe of Britain: insights from small mammal phylogeography. Proc Biol Sci (2009) 276:4287-94. doi:10.1098/rspb.2009.1422

42. Comstedt P, Asokliene L, Eliasson I, Olsen B, Wallensten A, Bunikis J, et al. Complex population structure of Lyme borreliosis group spirochete Borrelia garinii in subarctic eurasia. PLoS One (2009) 4:e5841. doi:10.1371/journal.pone. 0005841

43. Vennestrøm J, Egholm H, Jensen PM. Occurrence of multiple infections with different Borrelia burgdorferi genospecies in Danish Ixodes ricinus nymphs. Parasitol Int (2008) 57:32-7. doi:10.1016/j.parint.2007.07.004 
44. Brisson D, Dykhuizen DE. OspC diversity in Borrelia burgdorferi: different hosts are different niches. Genetics (2004) 168:713-22. doi:10.1534/genetics. 104.028738

45. Crowder CD, Matthews HE, Schutzer S, Rounds MA, Luft BJ, Nolte O, et al. Genotypic variation and mixtures of Lyme Borrelia in Ixodes ticks from North America and Europe. PLoS One (2010) 5:e10650. doi:10.1371/journal.pone. 0010650

46. Dykhuizen DE, Brisson D. Evolutionary genetics of Borrelia burgdorferi sensu lato. In: Samuels DS, Radolf JD, editors. Borrelia: Molecular Biology, Host Interaction and Pathogenesis. Norfolk: Caister Academic Press (2010). p. 221-49.

47. Bunikis J, Noppa L, Östberg Y, Barbour AG, Bergström S. Surface exposure and species specificity of an immunoreactive domain of a 66-kilodalton outer membrane protein (P66) of the Borrelia spp. that cause Lyme disease. Infect Immun (1996) 64:5111-6.

48. Dykhuizen DE, Baranton G. The implications of a low rate of horizontal transfer in Borrelia. Trends Microbiol (2001) 9:344-50. doi:10.1016/S0966-842X(01) 02066-2

49. Hanincová K, Ogden NH, Diuk-Wasser M, Pappas CJ, Iyer R, Fish D, et al. Fitness variation of Borrelia burgdorferi sensu stricto strains in mice. Appl Environ Microbiol (2008) 74:153-7. doi:10.1128/AEM.01567-07

50. Michalik J, Skotarczak B, Skoracki M, Wodecka B, Sikora B, Hofman T, et al. Borrelia burgdorferi sensu stricto in yellow-necked mice and feeding Ixodes ricinus ticks in a forest habitat of west central Poland. J Med Entomol (2005) 42:850-6. doi:10.1603/0022-2585(2005)042[0850:BBSSIY]2.0.CO;2

51. Humair P, Gern L. Relationship between Borrelia burgdorferi sensu lato species, red squirrels (Sciurus vulgaris) and Ixodes ricinus in enzootic areas in Switzerland. Acta Trop (1998) 69:213-27. doi:10.1016/S0001-706X(97) 00126-5

52. Lane RS, Mun J, Eisen RJ, Eisen L. Western gray squirrel (Rodentia: Sciuridae): a primary reservoir host of Borrelia burgdorferi in Californian oak woodlands? J Med Entomol (2005) 42:388-96. doi:10.1603/0022-2585(2005) 042[0388:WGSRSA]2.0.CO;2

Conflict of Interest Statement: The authors declare that the research was conducted in the absence of any commercial or financial relationships that could be construed as a potential conflict of interest.

Received: 10 July 2014; accepted: 13 August 2014; published online: 28 August 2014. Citation: James MC, Gilbert L, Bowman AS and Forbes KJ (2014) The heterogeneity, distribution, and environmental associations of Borrelia burgdorferi sensu lato, the agent of Lyme borreliosis, in Scotland. Front. Public Health 2:129. doi: 10.3389/fpubh.2014.00129

This article was submitted to Epidemiology, a section of the journal Frontiers in Public Health.

Copyright (C) 2014 James, Gilbert, Bowman and Forbes. This is an open-access article distributed under the terms of the Creative Commons Attribution License (CC BY). The use, distribution or reproduction in other forums is permitted, provided the original author(s) or licensor are credited and that the original publication in this journal is cited, in accordance with accepted academic practice. No use, distribution or reproduction is permitted which does not comply with these terms. 